\title{
Metabolic risk factor profile in patients on treatment with second generation antipsychotics
}

\author{
Zahan $\mathrm{T}^{1}$, Akhter $\mathrm{N}^{2}$, Mullick MSI${ }^{2}$, Dewan $\mathrm{ZF}^{2}$ \\ ${ }^{1}$ Department of Pharmacology, Holy Family Red Crescent Medical College, 1 Eskaton Road, Dhaka, \\ Bangladesh ${ }^{2}$ Department of Pharmacology Bangabandhu Sheikh Mujib Medical University (BSMMU), \\ Shahbagh, Dhaka-1000, Bangladesh \\ Email:tahminazahan@yahoo.com
}

\begin{abstract}
The second generation antipsychotic agents, although exhibit superior safety profile, is associated with metabolic adverse effects including weight gain, diabetes mellitus and hyperlipidaemia. These adverse effects are not only the risk factors for cardiovascular disease and diabetes mellitus but may also impair patient's adherence to treatment. However, different member of second generation antipsychotics differ in their extent of metabolic adverse effects. The aim of the study was to evaluate the association between olanzapine, risperidone or quetiapine treatment and body mass index, blood pressure, diabetes mellitus and hyperlipidaemia in patients with Schizophrenia and Bipolar Disorder. Forty-four cases of Schizophrenia and Bipolar Disorder diagnosed with DSM-IV criteria were selected according to inclusion and exclusion criteria. Body weight, body mass index and blood pressure were measured at baseline, at the end of 4th, 8th and 12th weeks of treatment. Blood samples were collected to measure blood glucose and serum lipid profile at baseline and at the end of 4th, 8th and 12th weeks in the study group receiving treatment (olanzapine $20-30 \mathrm{mg}$ /day, risperidone $4-16 \mathrm{mg} /$ day and quetiapine 300-800 $\mathrm{mg} /$ day) after overnight fasting. Therapeutic use of olanzapine and risperidone in Schizophrenia and Bipolar Disorder for a period of 4th, 8th and 12th weeks was associated with significant increase in body weight and body mass index. Quetiapine did not cause significant changes in body weight and body mass index after 4 and 8 weeks. However, after 12 weeks treatment, body mass index increased significantly. Olanzapine, risperidone and quetiapine increased the blood glucose level significantly after 8 and 12 weeks treatment. Olanzapine and risperidone elevated the serum cholesterol, triglyceride and low density lipoprotein levels significantly after 4,8 and 12 weeks. But quetiapine showed no significant change in lipid profile. However, olanzapine and risperidone significantly increased triglyceride level after 8 and 12 weeks. Amongst three drugs, quetiapine treatment increased high density lipoprotein level. Our study revealed that quetiapine treatment is associated with less risk of dyslipidaemia.
\end{abstract}

Key words: Weight gain, hyperglycaemia, dyslipidaemia, psychotic patients

\section{Introduction}

Mental illness has reached to an alarming proportion over the globe and has become a vitally important issue for the nations in terms of morbidity, mortality and huge economic burden. Schizophrenia is the most common psychotic disorder. It affects 24 million people across the world ${ }^{1}$. Premature mortality, associated with major mental disorders, is primarily related to coronary heart disease. Such patients have a 2-3 fold increased risk of death, and this mortality gap associated with mental illness compared to the general population has widened in recent decades..$^{2-3}$
Schizophrenia and Bipolar Disorder are associated with an increased risk for obesity related cardiovascular mortality. ${ }^{4}$ In psychosis there is reduced central serotoninergic neurotransmission that results in increased food intake and weight gain and can contribute to increased storage of fat (in visceral adipose tissue) which leads to dyslipidaemia, hypertension, and increased risk for Type 2 Diabetes Mellitus and is, therefore, a key factor in the development of cardiovascular disease and associated mortality and morbidity..$^{5-6}$ Framingham Heart study confirmed that in patients with obesity, diabetes, hypertension and dyslipidaemia, there is increased 
risk of cardiovascular disease and it has also been suggested to occur at a higher frequency in patients with Schizophrenia than in the general population (about 1.5 to 2 times greater). ${ }^{6-7}$

Two groups of antipsychotic drugs are available in the treatment of Schizophrenia and Bipolar Disorder: First generation antipsychotic (FGA) and Second generation antipsychotic (SGA) drugs. Chlorpromazine, haloperidol is the widely used FGAs. The primary disadvantage of firstgeneration antipsychotics is the lack of response to negative symptoms. Moreover, they show high rate of extrapyramidal side effects (EPSE). The superiority of the second generation or atypical agents like clozapine, olanzapine, quetiapine, risperidone, ziprasidone, palliperidone is most effective for reducing the risk of EPSE. For positive symptoms of schizophrenia, the effectiveness of these drugs appears to be comparable to that of older agents. ${ }^{8-10}$ Recently there has been considerable interest and concern about the metabolic abnormalities associated with SGAs use ${ }^{11}$. Antipsychotics can lead to increased appetite by interfering with the dopamine reward system. Other causes such as disruption of hypothalamic regulation of glucose levels may be responsible for medication induced diabetes. ${ }^{8}$ Transmission mediated by $\alpha$-adrenergic receptors seems to have an effect on stimulation of appetite. Blockade of $\mathrm{H}_{1}$ receptors is involved in increased appetite and consequent weight gain ${ }^{12}$. Several antipsychotics block histamine receptors, and there seems to be a logarithmic relationship between affinity for these receptors and stimulation of appetite. Olanzapine is the drug which has got the highest affinity for $\mathrm{H}_{1}$ receptor. In addition, blockade of serotoninergic $5 \mathrm{HT}_{2} \mathrm{C}$ receptors also leads too increased appetite. ${ }^{13-14}$

Abnormalities in lipid metabolism have been shown to occur in patients treated with clozapine, olanzapine, quetiapine, risperidone or haloperidol. Olanzapine has been associated with significant increase in serum triglyceride levels and severe hyperlipidaemia ${ }^{15}, 16$. According to a recent US consensus statement, the SGAs vary in their propensity to cause obesity, hyper glycaemia and hyperlipidaemia, with clozapine and olanzapine showing the greatest effects, risperidone and quetiapine having intermediate effects, and aripiprazole and ziprasidone have lowest effects.
Divergent results have been seen with risperidone and quetiapine. ${ }^{11}$

All the members of the second-generation drugs are not equally implicated to cause metabolic adverse effects. This study therefore, was designed to investigate the extent to which monotherapy of olanzapine, risperidone or quetiapine, widely used drugs in patients with Schizophrenia and Bipolar Disorder affects body weight, blood glucose level and lipid profile.

\section{Materials and Methods}

This was a prospective type of interventional study conducted in the Department of Psychiatry and Department of Pharmacology, (BSMMU) Dhaka, from October, 2011 to January, 2013 on 44 diagnosed cases of Schizophrenia and Bipolar Disorder. Ethical clearance was obtained from Institutional Review Board (IRB) of the University. Study design is shown in Fig 1.

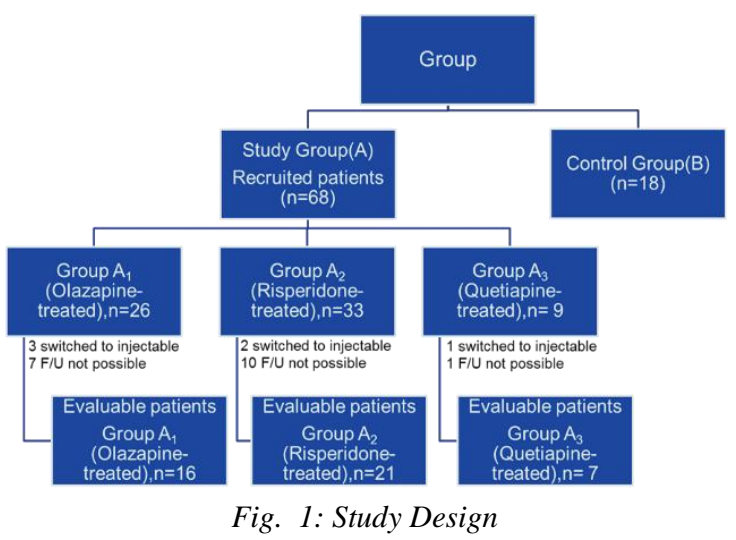

Patients with Schizophrenia and Bipolar Disorder of either sex with an age range between 18-50 years, diagnosed according to DSM-IV criteria taking either olanzapine, risperidone or quetiapine monotherapy were included in the study. Only patients who had a normal blood pressure, glucose level and lipid profile were recruited.

Patients with history of taking antipsychotic medications at least for last 30 days, those currently having diabetes mellitus, hypertension and hyperlipidaemia were excluded; Patients having serious cognitive deficit that causes disturbance of communication and associated serious infections or terminal illness were not included. 
Study Design: Study population was grouped as follows:

Group $A_{1}$ consisted of 26 patients, received olanzapine at a dose of 20-30 mg orally daily.

Group $\mathrm{A}_{2}$ comprised of 33 patients, received risperidone at a dose of 4-16 mg orally daily.

Group $\mathrm{A}_{3}$ consisted of 9 patients, received quetiapine at a dose of 300-800 mg orally daily.

All patients were treated with respective drugs for a period of 12 weeks.

Prior to study, written informed consent was taken from the patients or care- givers.

Blood collection: With all aseptic precaution $5 \mathrm{ml}$ blood was collected in $\mathrm{K}_{3}$ EDTA (Tri potassium Salt of Ethylene Diamine Tetra Acetate) containing test tube. Fasting blood samples of psychotic patients were collected once on admission into the hospital before starting antipsychotic medications (olanzapine, risperidone and quetiapine) to measure blood glucose \& serum lipid profile which were recorded as "baseline levels". Fasting blood samples were repeated again after 4,8 and 12 weeks, after antipsychotic medications.

BMI was measured by Flier and Flier equation:

$\mathrm{BMI}=\frac{\text { Weight in } \mathrm{kg}}{\text { Hight in } \mathrm{m}^{2}}$

Blood glucose, serum cholesterol, triglyceride, low density lipoprotein and high density lipoprotein was analyzed enzymatically using commercial kits.

\section{Statistical analysis:}

All analysis was carried out with the help of Statistical Package for Social Sciences (SPSS-17). Values of age, blood glucose level and lipid profile were fed in the SPSS. One Way ANOVA was done to compare the baseline body weight, BMI, blood glucose and serum lipid profile amongst the study group. Mean values of body weight, BMI, blood glucose and serum lipid profile for all the four groups were determined for baseline, after 4, 8 and 12 weeks, were compared between 4 groups by using Repeated Measure ANOVA analysis.

\section{Result}

The average age of the study group was $28.8 \pm 8.9$ years of which 29 were male and 15 were female. 32 patients were diagnosed as Schizophrenia and 12 patients as Bipolar Disorder. Family history of mental illness was positive for $34 \%$ patients.

Table I: Comparison of baseline body weight, BMI, blood glucose and lipid profile amongst three groups

\begin{tabular}{lc}
\hline Variables & P value \\
\hline Body weight $(\mathrm{kg})$ & 0.379 \\
BMI $\left(\mathrm{kg} / \mathrm{m}^{2}\right)$ & 0.241 \\
Blood glucose & 0.549 \\
Cholesterol & 0.608 \\
Triglyceride & 0.153 \\
LDL-C & 0.229 \\
HDL-C & 0.068 \\
\hline
\end{tabular}

ONE WAY ANOVA test was done to measure the level of significance

Table I show the comparison of different parameters amongst the three groups at baseline. There was no significant difference in any parameters.

Table II: Changes in Body weight and BMI in patients on antipsychotic medications

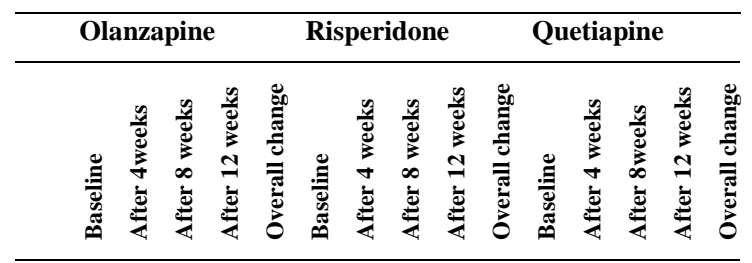
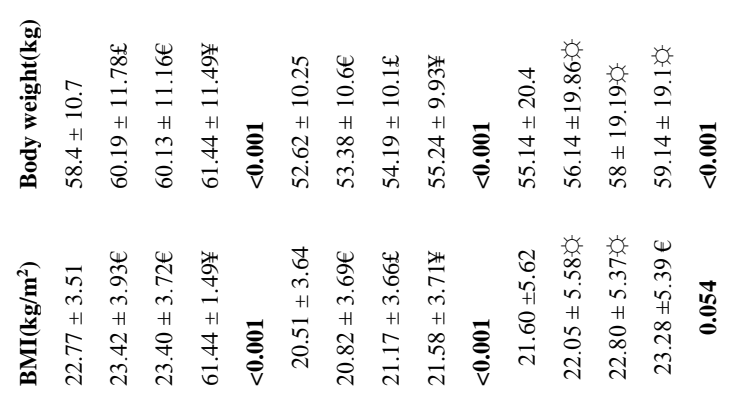

Values are expressed as mean \pm SD; REPEATED MEASURE ANOVA test was done to measure level of significance; $* * *$ significant at $\mathrm{p} \leq$ $0.001=¥ ; \mathrm{p} \leq 0.01=£ ; \mathrm{p} \leq 0.05=€ ; \mathrm{p} \geq 0.05=-{ }^{\prime}$, when the study group at the end of 4,8 and 12 weeks was compared

Table II illustrates the changes in body weight in different groups of patients, at different time interval. In olanzapine treated group, body weight increased significantly $(\mathrm{p}<0.05)$ after 4 weeks, $(\mathrm{p}$ $<0.05) 8$ weeks and $(\mathrm{p}<0.01) 12$ weeks; whereas overall change in body weight was significant ( $p$ $<0.001)$. While in risperidone treated group, body weight increased significantly $(\mathrm{p}<0.05)$ after 4 
weeks, $(\mathrm{p}<0.01) 8$ weeks and $(\mathrm{p}<0.001)$ after 12 weeks; whereas overall change in body weight was significant $(\mathrm{p}<0.001)$. While no significant change in body weight was observed after 4,8 and 12 weeks, in quetiapine treated group, as compared to baseline; whereas overall change in body weight was significant $(\mathrm{p}<0.001)$. Table II also shows the changes in BMI. In olanzapine treated group, significant change in BMI was observed after 4 weeks ( $\mathrm{p}<0.05), 8$ weeks ( $<$ $0.05)$ and 12 weeks $(\mathrm{p}<0.001)$; whereas overall change in BMI was significant $(\mathrm{p}<0.001)$. While in risperidone treated group, BMI increased significantly $(\mathrm{p}<0.05)$ after 4 weeks, $(\mathrm{p}<0.01) 8$ weeks and ( $p<0.001) 12$ weeks; whereas overall change in body weight was significant $(\mathrm{p}<0.001)$. In quetiapine treated group no significant change in BMI was observed after 4 and 8 weeks. However, it increased significantly $(\mathrm{p}<0.05)$ after 12 weeks, as compared to baseline. While overall change in BMI was not significant.

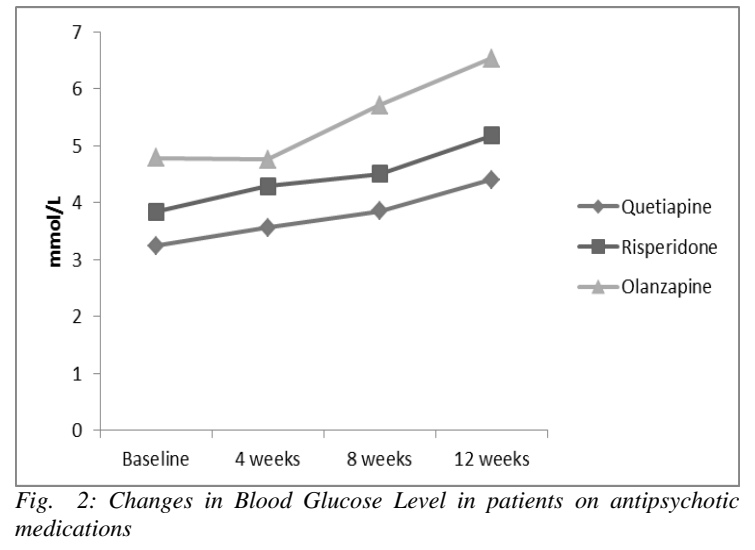

Fig 2 shows increase in blood glucose level was highest with olanzapine treatment, followed by risperidone and quetiapine treated groups.

Table-III shows the changes in lipid profile in patients. In olanzapine treated group, serum cholesterol level significantly increased $(\mathrm{p}<0.01)$ after 4 weeks, $(\mathrm{p}<0$.01) 8 weeks and $(\mathrm{p}<0.001)$ 12 weeks; whereas overall change in cholesterol was significant $(\mathrm{p}<0.001)$; In risperidone treated group, significant rise was observed after $4(\mathrm{p}<$ $0.01), 8$ ( $\mathrm{p}<0.01)$ and 12 weeks $(\mathrm{p}<0.001)$; whereas overall change in cholesterol was significant $(p<0.001)$. While in quetiapine treated group, no significant rise was observed after 4,8 and 12 weeks, as compared to baseline; whereas overall change in cholesterol level was not significant. Table IV also illustrates the changes in plasma TG level. In olanzapine treated group, TG level was significantly increased $(\mathrm{p}<0.001)$ after 4 weeks, $(\mathrm{p}<0.001) 8$ weeks and $(\mathrm{p}<0.001)$ 12 weeks; whereas overall change in TG was significant $(\mathrm{p}<0.001)$.

Table III: Changes in lipid profile in patients on antipsychotic medications

\begin{tabular}{|c|c|c|c|c|c|c|c|c|c|c|c|c|c|c|}
\hline & \multicolumn{5}{|c|}{ Olanzapine } & \multicolumn{4}{|c|}{ Risperidone } & \multicolumn{5}{|c|}{ Quetiapine } \\
\hline 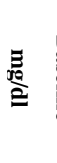 & 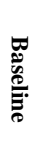 & 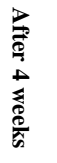 & & $\frac{8}{0}$ & 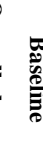 & 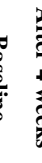 & 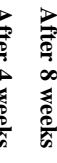 & 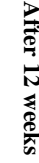 & & 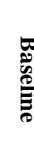 & & 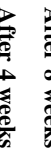 & & 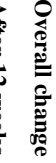 \\
\hline
\end{tabular}

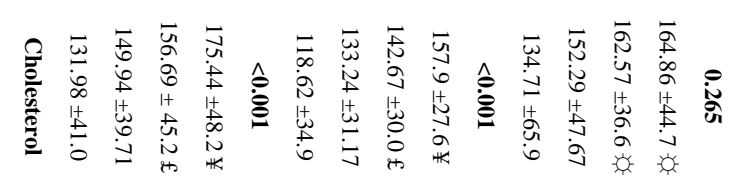

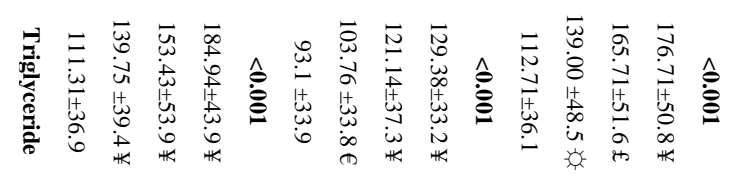

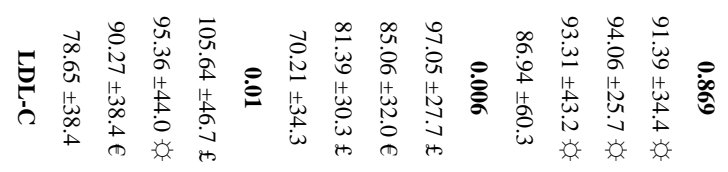

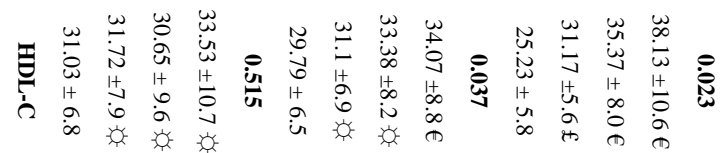

$\overline{\text { Values are expressed as mean } \pm \text { SD; REPEATED MEASURE ANOVA }}$ test was done to measure level of significance; $* * *$ significant at $\mathrm{p} \leq 0.001=$ $¥ ; \mathrm{p} \leq 0.01=£ ; \mathrm{p} \leq 0.05=€ ; \mathrm{p} \geq 0.05=$; , when the study group at the end of 4,8 and 12 weeks was compared

In risperidone treated group significant rise $(\mathrm{p}<$ $0.05)$ was observed after 4 weeks, $(p<0.001) 8$ weeks and $(p<0.001) 12$ weeks; whereas overall change in TG was significant $(\mathrm{p}<0.001)$. In quetiapine treated group no significant rise was seen after 4 weeks. However, significant rise $(\mathrm{p}<$ $0.01)$ was observed after 8 weeks and $(\mathrm{p}<0.001)$ 12 weeks; whereas overall change in TG was significant $(\mathrm{p}<0.001)$. Table-III shows the changes in serum LDL-C level. In olanzapine treated group, LDL-C level was significantly increased $(\mathrm{p}<0.05)$ after 4 weeks and $(\mathrm{p}<0.01)$ 12 weeks; whereas overall change in LDL-C was significant $(p<0.05)$. In risperidone treated group, LDL-level significantly increased $(\mathrm{p}<0.01)$ after 4 weeks, $(p<0.05) 8$ weeks and $(p<0.01) 12$ weeks; whereas overall change in LDL-C was significant $(\mathrm{p}<0.01)$. In quetiapine treated group, 
no significant rise in LDL-level was observed after 4,8 and 12 weeks of treatment; whereas overall change was not significant in LDL-C level. Table-IV illustrates the changes in serum HDL-C level. In olanzapine treated group, no significant change in serum HDL-C level was observed after treatment of any duration; whereas overall change in HDL-C level was not significant. In risperidone treated group, significant rise was observed $(\mathrm{p}<0.05)$ after 12 weeks; whereas overall change in HDL-C level was significant $(p<0.05)$. While in quetiapine treated group significant rise $(\mathrm{p}<0.01)$ was observed after 4 weeks, $(\mathrm{p}<0.05) 8$ weeks and $(\mathrm{p}$ $<0.05) 12$ weeks; whereas overall change in LDL$\mathrm{C}$ was significant $(\mathrm{p}<0.05)$

\section{Discussion}

Schizophrenia and Bipolar Disorders are lifeshortening illness with higher mortality rates. In recent decades, though SGAs are the drugs of first choice because of improvement in negative symptoms and lack of EPSE, they have certain effects on metabolic parameters like body weight, blood glucose and lipid profile, which may in turn increase the risk of morbidity due to cardiovascular diseases. Amongst the SGAs, divergent results have been found about the association of different members of SGAs and weight gain, hyperglycaemia and dyslipidaemia. In our country, no such study has been conducted to explore the association of metabolic adverse effects and use of SGAs. Therefore, the present study has been designed to assess the effects of olanzapine, risperidone and quetiapine on body weight, BMI, blood glucose and lipid profile in patients with Schizophrenia and Bipolar Disorder. Our study showed that after 4, 8 and 12 weeks of treatment with olanzapine or risperidone there was a significant rise in body weight and BMI from baseline. Whereas, quetiapine treatment caused significant rise in BMI after 12 weeks. However, whereas overall change in the effect on body weight and BMI was significant, in all the three groups with the exception of quetiapine on BMI. Consistent with this study, olanzapine treatment for 6 weeks in schizophrenic or schizoaffective disorder patients was found to cause significant increase in body weight and BMI. ${ }^{18}$ Clinically significant weight gain is defined as $\geq 7 \%$ increase in weight $(\mathrm{kg})$ from baseline. $^{20}$ In olanzapine-treated patients, clinically significant weight gain occurred faster in the olanzapine group after 12 weeks, 1 year and 2 years compared to the haloperidol group. ${ }^{20} \mathrm{In}$ our study, body weight was increased by $7.25 \%$, $5.21 \%$ and $4.98 \%$ in quetiapine, olanzapine and risperidone treated group respectively, after 12 weeks of treatment. The relative receptor affinity of SGAs for dopamine, serotonin and histamine receptors leading to increased eating and weight gain have been suggested to be an underlying cause. $^{8,12}$

In the present investigation, after 4 weeks treatment with risperidone and quetiapine there was significant rise in blood glucose level. After 8 and 12 weeks treatment all the three drugs significantly increased in blood glucose level; whereas overall change in blood glucose significant, in all the three groups. Several large population retrospective studies have found that olanzapine is associated with a significantly higher rate of diabetes than risperidone and quetiapine. ${ }^{22}$ In another randomized prospective study blood glucose level was found to be significantly elevated after 6 and 12 weeks of treatment with olanzapine but not with risperidone. ${ }^{19} \mathrm{~A}$ significant rise in blood glucose level was found with risperidone, in a crosssectional study. ${ }^{26}$ Discrepant results have been found about the association of quetiapine and hyperglycaemia. $^{23}$ Increased blood glucose with SGAs treatment could be related to the weight gain or obesity which can in turn cause insulin resistance ${ }^{17}$.

The present study showed that after 4 weeks of treatment, olanzapine or risperidone caused significant rise in cholesterol, TG and LDL-C levels. The rise in cholesterol and TG was significant with both drugs after 8 weeks of treatment. However, risperidone also caused significant rise in LDL-C level after 8 weeks. 12 weeks' treatment with olanzapine or risperidone caused significant rise in cholesterol, TG and LDL-C levels; quetiapine did not cause any significant change in those parameters after 4 weeks of treatment. After 8 and 12 weeks' treatment with quetiapine only TG level was increased significantly. However, overall change in TG level was observed in all the three groups. Whereas, overall change in the effect on cholesterol, TG and LDL-C level was significant, in olanzapine and risperidone treated group. 
Change in HDL-C level with olanzapine was not significant at any duration of treatment. While risperidone increased HDL-C level significantly after 12 weeks. Interestingly, quetiapine caused significant rise in HDL-C levels after 4, 8 and 12 weeks of treatment. Whereas, overall change in the effect on HDL-C level, in risperidone and quetiapine treated group was significant. However, HDL-C levels in patients of all the groups treated with olanzapine, risperidone and quetiapine was below $40 \mathrm{mg} / \mathrm{dl}$. Our findings regarding lipid profile are consistent with many other studies. In a randomized study that compared the atypical and typical antipsychotics, olanzapine was associated with significantly elevated mean cholesterol level after 6 weeks of treatment. $^{23}$ In a 14-week study, change in total cholesterol was assessed in 157 patients randomized to clozapine, olanzapine, risperidone, or haloperidol. There was a significant increase in mean total cholesterol at 8 and 14 weeks from baseline in case of olanzapine compared to risperidone. ${ }^{11}$ Our study showed olanzapine significantly elevated cholesterol after 12 weeks. It was shown that risperidone treated group had a significant increase in cholesterol level after 6 weeks compared with clozapine. ${ }^{16}$ In our study, cholesterol level increased significantly after 12 weeks in patients treated with risperidone. Divergent results have been found about the quetiapine induced hyperlipidaemia. ${ }^{11}$ The present study showed that mean TG level increased to $66 \%$ in olanzapine treated group after 12 weeks. The findings are consistent with openlabel and retrospective data demonstrating a greater association of olanzapine and clozapine treatment than risperidone with increases in cholesterol and triglycerides; ${ }^{25}$ Significantly higher LDL-C levels in risperidone treated group was found compared to clozapine. ${ }^{16}$ Dyslipidaemia caused by SGAs in this study could be related to insulin resistance developing after weight gain and is associated with dyslipidaemic triad, "an increase in TG and LDL-C and a decrease in HDL-C" ${ }^{26}$

Divergent results regarding hyperglycaemia and dyslipidaemia with the use of different members of SGAs could be due to variation in sample size, duration of treatment, severity of disease, race, social class and extent of weight gain.

Conclusion: The study concluded that quetiapine treatment is associated with low risk of dyslipidaemia, compared to olanzapine and risperidone. Further broad-based study is needed to confirm this finding.

\section{References}

1. http://www.who.int/mental_health/management / schizophrenia/en/ 11.17.2011

2. Colton CW, Manderscheid RW. Congruencies in increased mortality rates, years of potential life lost, and cause of death among public mental health clients in eight states. PrevChron Dis 2006; 3:42.

3. Saha S, Chant D, Mcgrath J. A systematic review of mortality in schizophrenia: is the mortality gap worsening over time. Arch Gen Psychiatry 2007; 64: 1123-1131.

4. Newcomer JW, Campos JA, Marcus RN, Breder C, Berman RM, Kerseaer W, L'Italein, Nys M, Carson WH, Mcquade RD. A multicenter, randomized, double-blind study of the effects of aripiprazole in overweight subjects with schizophrenia or schizoaffective disorder switched from olanzapine. J Clin Psychiatry 2008; 69: 46-56.

5. Zhang ZJ, Yao ZJ, Liu W. Effects of antipsychotics on fat deposition on fat deposition and changes in leptin and insulin levels. Magnetic resonance imaging study of previously untreated people with schizophrenia. Br J Psychiatrty 2004; 184: 58-62.

6. Khot UN, Khot MB, Bajzer CT, Sapp SK, Ohman EM, Brener SJ, Ellis SC, Lincoff AM, Topol EJ. Prevalence of conventional risk factors in patients with coronary heart disease. JAMA 2003; 290: 7.

7. Ryan MC, Thakore JH. Physical consequences of schizophrenia and its treatment. the metabolic syndrome. Life Sci 2002; 71: 39-57.

8. Llorente MD, Urrutia V. Diabetes, psychiatric disorders, and the metabolic effects of antipsychotic medications. Clin Diabetes 2006; 24: $18-24$.

9. Gardner DM, Baldessarini RJ, Waraich P. Modern antipsychotic drugs: a critical overview. Can Med Asso Journal 2005; 172: 1703-1711.

10. Lieberman JA, Stroup TS, McEvoy JP, Swartz MS, Rosenheck RA, Perkins DO, Keefe RS, Davis SM, Davis CE, Lebowitz BD, Severe J, Hsiao JK. The Clinical Antipsychotic Trials of Intervention Effectiveness (CATIE) Investigators: Effectiveness of antipsychotic drugs in patients with chronic schizophrenia. $\mathrm{N}$ Engl J Med 2005; 353: 9-23.

11. American Diabetes Association; American Psychiatric Association; American Association of Clinical Endocrinologists; North American Association for the Study of Obesity Consensus development conference on antipsychotic drugs and obesity and diabetes. Diabet care 2004; 7 14. 
12. Zimmermann U, Kraus T, Himmerich H, Schuld A, Pollmächer T. Epidemiology, implications and mechanisms underlying drug-induced weight gain in psychiatric patients.J Psychiatr Res 2003; 37: 193-220.

13. Berg J, Stajich G, Zdanowicz M. Atypical antipsychotic-induced type 2 diabetes. Pharmacy Times 2012.

14. Holt RIG, Peveler RC, Byrne CD. Schizophrenia, the metabolic syndrome and diabetes. Diabet Medicine 2004; 21: 515-523.

15. Lindenmeyer JP, Czober P, Volavka J, Citrome L, Sheitman B, Mcevoy JP, Cooper TB, Chakos M, Lieberman JA. Changes in glucose and cholesterol levels in patients with typical or atypical antipsychotics. Am J Psychiatry 2003; 160: 290-296.

16. Idonije OB, Festus OO, Akpamu U, Okkhiai, O, Iribhogbe OI, Ialomhe GBS. A comparative study of the effects of clozapine and risperidone monotherapy on lipid profile in Nigerian patients with schizophrenia. Science Alert2012; 8: 27-34.

17. Bergman RN, Ader M. Free fatty acids and pathogenesis of type 2 diabetes mellitus. Trends EndocrinolMetabol 2000; 11: 351-356.

18. Simpson GM, Glick ID, Weiden PJ, Romano SJ, Siu CO. Randomized, controlled, doubleblind multicenter comparison of the efficacy and tolerability of ziprasidone and olanzapine in acutely ill inpatients with schizophrenia or schizoaffective disorder. Am J Psychiatry 2004; 161: 37-47.

19. Ignole S, Belorkar NR, Waradkar P, Shrivastava M. Comparison of effects of olanzapine and Risperidone on body mass index and blood sugar level in schizophrenic patients. Indian J PhysiolPharmacol 2009; 53: 47-54.
20. Alveraz-Jimennez M, Gonzalez-Blanch C, Vazquez-Barquero. Attenuation of antipsychotic-induced weight gain with early behavioral intervention in drug-naïve firstepisode psychosis patients: a randomized controlled trial. J Clin Psychiatry 2006; 67: 5360 .

21. Sernyak MJ, Leslie DL, Alarcon RD, Losonczy MF, Rosenheck R. Association of diabetes mellitus with use of atypical neuroleptics in the treatment of schizophrenia. Am J Psychiatry 2002; 159: 561-566.

22. Buse JB, Cavajjoni P, Hornbuckle K, Hutchins D, Breier A, Jovanonic L. A retrospective cohort study of diabetes mellitus and antipsychotic treatment in the U.S.J ClinEpidemiol 2003; 56: 164-170.

23. Akhtar N, Rana MH. Conventional and atypical antipsychotics; changes in glucose and cholesterol levels. Prof Med J 2012; 19: 93-97.

24. Wirshing DA, Boyd JA, Meng LR. The effects of novel antipsychotics on glucose and lipid levels. J Clin Psychiatry 2002; 63: 856-865.

25. Meyer JM. A retrospective comparison of lipid, glucose and weight changes at one year between olanzapine and risperidone treated in patients, in Proceedings of $39^{\text {th }}$ Annual Meeting of the American College of Neuropsychopharmacology' 2000, Nashville Tenn, ACNP, vol. pp. 425-433.

26. Smith DA. Treatment of the Dyslipidaemia of insulin resistance.Med Clin N America.2007; 91: 1185-1210. 